\title{
Algebraic method for the harmonic oscillator with a minimal length
}

\author{
P. Valtancoli \\ Dipartimento di Fisica, Polo Scientifico Universitá di Firenze \\ and INFN, Sezione di Firenze (Italy) \\ Via G. Sansone 1, 50019 Sesto Fiorentino, Italy
}

\begin{abstract}
We show that the algebraic method solving the ordinary harmonic oscillator can be adapted to the non-commutative case.
\end{abstract}




\section{Introduction}

It has been suggested that a new interesting structure at short distances, characterized by a finite minimal position uncertainty $\Delta x_{0}$, can be modeled with a small modification of the commutation rules. It is also expected by plausibility arguments of string theory that such a short distance behaviour emerges at the Planck scale.

Moreover there are many examples from other physical problems involving quanta that cannot be localized to a point, i.e. quasi-particles and collective excitations. The most elementary example of a non point-like particle is the harmonic oscillator with a minimal length. This problem has been successfully solved in [1], using the momentum representation. In 2] it has been discussed that for this case it is not possible to define generalized raising and lowering operators.

It is aim of this article to show instead that it is possible to solve this non-commutative problem with a totally algebraic method, based only on the modified commutation rule

$$
[\hat{x}, \hat{p}]=i \hbar\left(1+\beta \hat{p}^{2}\right)
$$

This allows us to represent the excited states by applying a string of operators to the ground state, as one usually does in the standard case. This representation is useful to solve the eigenvalue problem, compute all the normalizations and the expectation values of the operator $\hat{x}^{2}$ on the excited states.

\section{Ladder operators}

In literature it has been solved the quantization of the harmonic oscillator

$$
\hat{H}=\frac{1}{2 m}\left(\hat{p}^{2}+m^{2} \omega^{2} \hat{x}^{2}\right)
$$

in presence of the commutation rule

$$
[\hat{x}, \hat{p}]=i \hbar\left(1+\beta \hat{p}^{2}\right)
$$

The method employed is finding the eigenvalues and eigenvectors of the Hamiltonian

(2.1) in the momentum representation. Instead in the present article we want to solve the same problem using purely algebraic manipulations, based only on the commutation rule (2.2). 
In the case of the standard harmonic oscillator the quantization leads to equally spaced energy levels. Therefore it is possible to shift from one energy level to another by adding or removing a certain amount of energy quanta. This concept is practically realized by moving from the operators $\hat{x}$ and $\hat{p}$ to two news operators factorizing the Hamiltonian. If $\hat{x}$ and $\hat{p}$ were commuting, then the Hamiltonian could be written as the product of two factors:

$$
\frac{1}{2 m}(-i \hat{p}+m \omega \hat{x})(i \hat{p}+m \omega \hat{x})
$$

In presence of the standard commutation rule of quantum mechanics we can still write the Hamiltonian in a factorized form apart from a constant:

$$
\hat{H}=\hbar \omega\left(a^{\dagger} a+\frac{1}{2}\right)
$$

where we define the ladder operator

$$
a=\frac{m \omega \hat{x}+i \hat{p}}{\sqrt{2 m \hbar \omega}}
$$

In presence of the more complex commutation rule (2.2), it is intriguing that it is again possible to write the Hamiltonian in a factorized form apart from a constant

$$
\hat{H}=\frac{\hbar \omega}{2}\left[\frac{a^{\dagger}(\mu) a(\mu)+\mu}{\sqrt{\mu(\mu-1)}}\right]
$$

where

$$
a(\mu)=\frac{\hat{x}+1 \hbar \beta \mu \hat{p}}{\hbar \sqrt{\beta}} \quad \mu=\frac{1}{2}+\frac{1}{2} \sqrt{1+\frac{4}{m^{2} \omega^{2} \hbar^{2} \beta^{2}}}
$$

and the parameter $\mu$ is fixed from the commutation rule (2.2) and from the form of the Hamiltonian (2.1). This factorization will be useful in the following to describe the construction of the ground state.

In the limit $\beta \rightarrow 0$ i.e. $\mu \rightarrow \infty$

$$
a(\mu) \rightarrow \sqrt{2 \mu} a
$$

where $a$ is the typical ladder operator of the harmonic oscillator, and the Hamiltonian reduces to the standard form 


$$
H \rightarrow \hbar \omega\left(a^{\dagger} a+\frac{1}{2}\right)
$$

\section{Commutation rules}

To show that the ladder operators $a(\mu), a^{\dagger}(\mu)$ are useful to raise or lower the number of energy quanta it is necessary to study the commutation rule between $a(\mu)$ and $a^{\dagger}(\mu)$. In the case of the standard harmonic oscillator it is well known that

$$
\left[a, a^{\dagger}\right]=1
$$

Unfortunately in our case the operators $a(\mu), a^{\dagger}(\mu)$ do not form any closed algebra, however by carefully manipulating the possible combinations between $\hat{x}$ and $\hat{p}$ we have been able to derive the following meaningful rule

$$
a(\mu) a^{\dagger}(\mu)-a^{\dagger}(\mu+1) a(\mu+1)=1+2 \mu
$$

We now apply the Hamiltonian operator in the form (2.6) to $a^{\dagger}(\mu)$

$$
H a^{\dagger}(\mu)=\frac{\hbar \omega}{2}\left[\frac{a^{\dagger}(\mu) a^{\dagger}(\mu+1) a(\mu+1)+(1+3 \mu) a^{\dagger}(\mu)}{\sqrt{\mu(\mu-1)}}\right]
$$

This formula will be useful to discuss the construction of all higher energy states.

\section{Spectrum of the Hamiltonian}

By applying the factorized form of the Hamiltonian (2.6) to a generic state $\psi(p)$ we note that the mean value of energy reaches its minimum with the choice

$$
a(\mu) \psi_{0}(\mu)=0
$$

Since the operator $\hat{x}$ is described in the momentum representation by

$$
\hat{x}=i \hbar\left(1+\beta p^{2}\right) \frac{\partial}{\partial p}
$$

the eigenfunction of the ground state is given by 


$$
\psi_{0}(\mu)=\left(\sqrt{\frac{\beta}{\pi}} \frac{\Gamma(\mu+1)}{\Gamma\left(\mu+\frac{1}{2}\right)}\right)^{\frac{1}{2}}\left(1+\beta p^{2}\right)^{-\frac{\mu}{2}}
$$

and the corresponding eigenvalue is

$$
E_{0}=\frac{\hbar \omega}{2} \sqrt{\frac{\mu}{\mu-1}}
$$

To build the first excited state it is enough to look at the equation (3.3). To cancel the first contribution it is convenient to apply this relation to $\psi_{0}(\mu+1)$

$$
\begin{aligned}
a(\mu+1) \psi_{0}(\mu+1) & =0 \\
H a^{\dagger}(\mu) \psi_{0}(\mu+1) & =\frac{\hbar \omega}{2} \frac{1+3 \mu}{\sqrt{\mu(\mu-1)}} a^{\dagger}(\mu) \psi_{0}(\mu+1)
\end{aligned}
$$

This formula allows us to define the first excited state as

$$
\psi_{1}(\mu)=c_{1}(\mu) a^{\dagger}(\mu) \psi_{0}(\mu+1)
$$

with eigenvalue

$$
E_{1}=\frac{\hbar \omega}{2} \frac{1+3 \mu}{\sqrt{\mu(\mu-1)}}
$$

Given the structure of the commutation rule (3.2) it is clear how to generalize to the case of the level $n$ excited state

$$
\begin{aligned}
\psi_{n}(\mu) & =c_{n}(\mu) a^{\dagger}(\mu) a^{\dagger}(\mu+1) \ldots a^{\dagger}(\mu+n-1) \psi_{0}(\mu+n)= \\
& =\frac{c_{n}(\mu)}{c_{n-1}(\mu+1)} a^{\dagger}(\mu) \psi_{n-1}(\mu+1)
\end{aligned}
$$

This state satisfies to the condition

$$
a(\mu+n) \ldots a(\mu+1) a(\mu) \psi_{n}(\mu)=0
$$

It is easy to show that these states are all eigenvectors of the Hamiltonian (2.6). By induction supposing that 


$$
a^{\dagger}(\mu) a(\mu) \psi_{n}(\mu)=f(n, \mu) \psi_{n}(\mu),
$$

using the definition of the generic excited state (4.8) and applying the rule (3.2) we obtain the recurrence equation

$$
f(n, \mu)=f(n-1, \mu+1)+1+2 \mu
$$

which is solved by

$$
f(n, \mu)=\sum_{i=0}^{n-1}(1+2(\mu+i))=n^{2}+2 n \mu
$$

Therefore we conclude that the energy eigenvalues of the generic $n$-eigenstate are

$$
E_{n}(\mu)=\frac{\hbar \omega}{2}\left(\frac{n^{2}+2 n \mu+\mu}{\sqrt{\mu(\mu-1)}}\right) \quad \rightarrow \quad \hbar \omega\left(n+\frac{1}{2}\right) \quad \text { for } \mu \rightarrow \infty
$$

We have still to compute the undetermined coefficients $c_{n}(\mu)$. By imposing the orthogonality condition and applying several times the rule (3.2) we obtain:

$$
c_{n}(\mu)=\left\{\prod_{k=0}^{n-1}\left[(n-k)^{2}+2(\mu+k)(n-k)\right]\right\}^{-\frac{1}{2}}
$$

and finally

$$
\frac{c_{n}(\mu)}{c_{n-1}(\mu+1)}=\frac{1}{\sqrt{n^{2}+2 n \mu}}
$$

\section{Comparison with the standard method}

The direct solution of the eigenvalue problem [1]-[3] gives rise to the following polynomial

$$
{ }_{2} F_{1}\left(-n, 2 s+1-n, s+1-n, \frac{1+i \sqrt{\beta} p}{2}\right)
$$

with the parameter $s=-\mu$. It is well known in literature that these are nothing else than Jacobi polynomials 


$$
{ }_{2} F_{1}(-n, \alpha+1+\beta+n, \alpha+1 ; x)=\frac{n !}{(\alpha+1)_{n}} P_{n}^{\alpha, \beta}(1-2 x)
$$

defined through the following formula

$P_{n}^{\alpha, \beta}(z)=\frac{\Gamma(\alpha+n+1)}{n ! \Gamma(\alpha+\beta+n+1)} \sum_{m=0}^{n}\left(\begin{array}{c}n \\ m\end{array}\right) \frac{\Gamma(\alpha+\beta+n+m+1)}{\Gamma(\alpha+m+1)}\left(\frac{z-1}{2}\right)^{m}$

They satisfy to the orthogonality condition:

$$
\int_{-1}^{1} P_{m}^{(\alpha, \beta)}(x) P_{n}^{(\alpha, \beta)}(x)(1-x)^{\alpha}(1+x)^{\beta} d x=c_{n} \delta_{n, m}
$$

where the coefficients $c_{n}$ are given by

$$
c_{n}=\frac{2^{\alpha+\beta+1}}{2 n+\alpha+\beta+1} \frac{\Gamma(n+\alpha+1) \Gamma(n+\beta+1)}{n ! \Gamma(n+\alpha+\beta+1)}
$$

The first polynomials are

$$
\begin{aligned}
P_{0}^{\alpha, \beta}(x) & =1 \\
P_{1}^{\alpha, \beta}(x) & =\frac{1}{2}[2(\alpha+1)+(\alpha+\beta+2)(x-1)] \\
P_{2}^{\alpha, \beta}(x) & =\frac{1}{8}[4(\alpha+1)(\alpha+2)+4(\alpha+\beta+3)(\alpha+2)(x-1)+ \\
& \left.+(\alpha+\beta+3)(\alpha+\beta+4)(x-1)^{2}\right]
\end{aligned}
$$

In our problem we notice that the parameters $\alpha$ and $\beta$ are fixed to be equal to

$$
\alpha=\beta=-\mu-n
$$

For example the wave function of the second excited state is proportional to

$$
P_{2}^{(-\mu-2,-\mu-2)}(-i \sqrt{\beta} p) \propto\left(1-(1+2 \mu) \beta p^{2}\right)
$$

Comparing with our method we can give the representation of this state as

$$
\psi_{2}(\mu) \sim a^{\dagger}(\mu) \psi_{1}(\mu+1) \sim a^{\dagger}(\mu) p\left(1+\beta p^{2}\right)^{-\frac{\mu+2}{2}}
$$


obtaining exactly the same polynomial defined above.

\section{Computation of the expectations values}

Since the excited states are obtained applying the string of operators $a^{\dagger}(\mu)$ that have definite parity with respect to the exchange $p \rightarrow-p$, we suddenly observe that the following expectation values

$$
<\psi_{n}(\mu)|\hat{x}| \psi_{n}(\mu)>=<\psi_{n}(\mu)|\hat{p}| \psi_{n}(\mu)>=0
$$

are zero as obtained with the standard quantization.

The more involved case is the expectation value of the operator $\hat{x}^{2}$. To reach this aim we observe that

$$
\begin{aligned}
\mid \psi_{n}(\mu)> & =\frac{a^{\dagger}(\mu)}{\sqrt{n^{2}+2 n \mu}} \mid \psi_{n-1}(\mu+1)> \\
\hat{x} \mid \psi_{n}(\mu)> & \left.=\frac{\hbar \sqrt{\beta}}{2}\left[a^{\dagger}(\mu)\left|\psi_{n}(\mu)>+\sqrt{n^{2}+2 n \mu}\right| \psi_{n-1}(\mu+1)\right)>\right]
\end{aligned}
$$

and the matrix elements of $\hat{x}^{2}$ is

$$
\begin{aligned}
<\psi_{n}(\mu)\left|\hat{x}^{2}\right| \psi_{n}(\mu)> & =\frac{\hbar^{2} \beta}{4}\left[\psi_{n}(\mu)\left|a(\mu) a^{\dagger}(\mu)\right| \psi_{n}(\mu)>+\right. \\
& +<\psi_{n-1}(\mu+1)\left|a^{2}(\mu)+a^{\dagger 2}(\mu)\right| \psi_{n-1}(\mu+1)>+ \\
& \left.+\left(n^{2}+2 n \mu\right)\right]
\end{aligned}
$$

Since

$$
\begin{aligned}
\frac{\hbar^{2} \beta}{4} a(\mu) a^{\dagger}(\mu) & =\frac{1}{4}\left(\hat{x}^{2}+\hbar^{2} \beta^{2} \mu(\mu+1) \hat{p}^{2}+\hbar^{2} \beta \mu\right) \\
\frac{\hbar^{2} \beta}{4}\left(a^{2}(\mu)+a^{\dagger 2}(\mu)\right) & =\frac{1}{2}\left(\hat{x}^{2}-\hbar^{2} \beta^{2} \mu^{2} \hat{p}^{2}\right),
\end{aligned}
$$

defining

$$
<\psi_{n}(\mu)\left|\hat{x}^{2}\right| \psi_{n}(\mu)>=f(n, \mu) \quad<\psi_{n}(\mu)\left|\hat{p}^{2}\right| \psi_{n}(\mu)>=g(n, \mu)
$$


we arrive at the conclusion that

$$
\begin{aligned}
3 f(n, \mu) & =\hbar^{2} \beta^{2} \mu(\mu+1) g(n, \mu)+2\left[f(n-1, \mu+1)-\hbar^{2} \beta^{2} \mu^{2} g(n-1, \mu+1)\right]+ \\
& +\hbar^{2} \beta\left(n^{2}+2 n \mu+\mu\right)
\end{aligned}
$$

We can add to this equation the information due to the eigenvalue equation

$$
f(n, \mu)+\hbar^{2} \beta^{2} \mu(\mu-1) g(n, \mu)=\hbar^{2} \beta\left(n^{2}+2 n \mu+\mu\right)
$$

By taking the difference between the last two equations we obtain

$$
f(n, \mu)=\hbar^{2} \beta^{2} \mu^{2} g(n, \mu)+f(n-1, \mu+1)-\hbar^{2} \beta^{2} \mu^{2} g(n-1, \mu+1)
$$

that, together with the eigenvalue equation (6.7), gives rise to the recurrence equation

$$
g(n, \mu)=\frac{2 \mu+1}{2 \mu-1} g(n-1, \mu+1)+\frac{2}{\beta(2 \mu-1)}
$$

This is solved simply by the following result

$$
\begin{aligned}
& g(n, \mu)=\frac{2 n+1}{\beta(2 \mu-1)} \\
& f(n, \mu)=\hbar^{2} \beta\left(n^{2}+(2 n+1) \frac{\mu^{2}}{2 \mu-1}\right)
\end{aligned}
$$

As you can see, many of the manipulations that one usually does with the ladder operators can be extended to the rule (3.2).

As a final note we can add the construction of the coherent states. These can be defined as the eigenfunctions of the ladder operator $a(\mu)$ :

$$
a(\mu) \psi_{\lambda}(\mu)=\frac{\lambda}{\hbar \sqrt{\beta}} \psi_{\lambda}(\mu)
$$

This equation is solved by

$$
\psi_{\lambda}(\mu) \sim\left(1+\beta p^{2}\right)^{-\frac{\mu}{2}} e^{-\frac{i \lambda}{\hbar \sqrt{\beta}} \arctan \sqrt{\beta p^{2}}}
$$

These states are analogous to the maximally localized states studied in [1]. 


\section{Conclusions}

We have learned from this article that it is possible in a non-commutative geometry to represent the eigenfunctions of the harmonic oscillator in a operatorial form. The major obstacle we have found is that the commutation rules between the raising and lowering operators are non-standard, i.e. they do not form a closed algebra. However one can still perform many of the manipulations of quantum mechanics like reproducing the results obtained with the differential equation method and computing the expectation values of the operators $\hat{x}^{2}$ e $\hat{p}^{2}$. Moreover it is possible define a non-commutative version of the coherent states, that look like the maximally localized states studied in [1].

Finally we have tried to generalize this operatorial method to the quantization of many harmonic oscillators with a minimal length [4]-[5], finding no interesting structure. However we will discuss this problem in detail in a forthcoming paper.

\section{References}

[1] A. Kempf, G. Mangano, R. B. Mann, Phys. Rev. D 52 (1995) 1108, hep-th/9412167.

[2] A. Bose, arXiv:1011.5234v1[physics.gen-ph].

[3] P. Valtancoli, Mod.Phys.Lett. A27 (2012) 1250107, arXiv:1205.3546.

[4] A. Kempf, J.Phys. A30 (1997) 2093, hep-th/9604045.

[5] F. Brau, J.Phys. A32 (1999) 7691, quant-ph/9905033v1. 